\title{
Deterministic preparation of Dicke states of donor nuclear spins in silicon by cooperative pumping
}

\author{
Yu Luo, Hongyi Yu, and Wang Yao* \\ Department of Physics and Center of Theoretical and Computational Physics, The University of Hong Kong, Hong Kong, China
}

(Received 10 October 2011; revised manuscript received 20 March 2012; published 5 April 2012)

\begin{abstract}
For donor nuclear spins in silicon, we show how to deterministically prepare various symmetric and asymmetric Dicke states which span a complete basis of the many-body Hilbert space. The state preparation is realized by cooperative pumping of nuclear spins by coupled donor electrons, and the required controls are in situ to the prototype Kane proposal for quantum computation. This scheme only requires a subgigahertz donor exchange coupling, which can be readily achieved without atomically precise donor placement, and hence it offers a practical way to prepare multipartite entanglement of spins in silicon with current technology. All desired Dicke states appear as the steady state under various pumping scenarios, and therefore the preparation is robust and does not require accurate temporal controls. Numerical simulations with realistic parameters show that Dicke states of 10-20 qubits can be prepared with high fidelity in the presence of decoherence and unwanted dynamics.
\end{abstract}

DOI: 10.1103/PhysRevB.85.155304

PACS number(s): 03.67.Bg, 42.50.Dv, 71.55.Cn, 76.70.Fz

\section{INTRODUCTION}

Because of the ultralong quantum coherence time, electron and nuclear spins of shallow donors in silicon are ideal candidates as information carriers in quantum computation. For a ${ }^{31} \mathrm{P}$ donor in isotope purified ${ }^{28} \mathrm{Si}$, a nuclear-spin coherence time exceeding $1 \mathrm{~s}$ and an electron spin coherence time exceeding $10 \mathrm{~ms}$ were measured. ${ }^{1-3}$ In his seminal paper, ${ }^{4}$ Kane proposed an architecture for scalable quantum computation using nuclear spins of gated ${ }^{31} \mathrm{P}$ donors in silicon as qubits. With superb compatibility with the existing silicon technology, Kane's proposal and its variants have stimulated extensive interest in donor systems in silicon, ${ }^{5-8}$ and remarkable technological progress has since been made in various aspects. Accurate positioning of the ${ }^{31} \mathrm{P}$ donor was made possible by controlled single-ion implantation. ${ }^{9}$ Local electrical tuning of the hyperfine interaction between donor electron and nuclear spins was demonstrated. ${ }^{10,11}$ An architecture that integrates a single ${ }^{31} \mathrm{P}$ donor with a silicon single electron transistor (SET) was recently developed which enables a high-sensitivity readout and control of the donor electron spin. ${ }^{12,13}$ In the meantime, challenges still remain, e.g., on realizing efficient donor electron exchange coupling which is also needed for mediating pairwise interaction of nuclear spins. ${ }^{14}$ Due to the interference between the degenerate valleys of the electron, the exchange coupling strength vastly oscillates with subnanometer variation in the donor position (e.g., between 1 and $100 \mathrm{GHz}$ when donor separation is $\sim 10 \mathrm{~nm}){ }^{14}$ The chance to have an exchange coupling of $\geqslant 30 \mathrm{GHz}$ expected by the initial Kane proposal becomes random unless with atomically precise dopant placement, ${ }^{15}$ which is beyond the technological capability today and in the near future.

In this paper, we introduce a scheme to deterministically prepare Dicke states of donor nuclear spins in silicon. Dicke states in general refer to the common eigenstates of $\hat{J}^{2}$ and $\hat{J}^{z}$ with eigenvalues $J(J+1)$ and $M$, respectively, where $\hat{J}$ is the collective spin of an ensemble of $N$ spin- $I$ particles. Dicke states with $J$ taking the maximum value $N I$ are symmetric under permutation operations. When $M \neq \pm J$, symmetric Dicke states are a class of genuine multipartite entangled states which have been widely pursued in atomic and optical systems as important resources for quantum information processing. ${ }^{16-24}$ These states have the remarkable properties that the entanglement is robust against qubit loss and projective measurements on the states lead to various entangled states of a lower qubit number. ${ }^{18}$ When $J$ takes a value other than $N I$, the Dicke states are no longer symmetric under all permutation operations and will be referred to here as asymmetric. Asymmetric Dicke states are also resources of genuine multipartite entanglement which are less studied for the lack of preparation schemes. The ideal resource for the optimal quantum telecoloning algorithm is one such state with $J=0 .{ }^{25}$ Preparation of asymmetric Dicke states also makes it possible to access the decoherence free subsystems in the presence of collective decoherence. ${ }^{26}$ The scheme we propose here can deterministically access all Dicke states (symmetric and asymmetric) that span a complete basis for the Hilbert space of $N$ spins. This direct access to the Dicke-states basis can be an important complement to the circuit model quantum information processing, since the entanglement of these collective states cannot be achieved in a simple way by pairwise interaction. ${ }^{18}$

Our scheme is based on cooperative pumping of nuclear spins by the coupled donor electrons, and the required controls are in situ to the prototype Kane proposal: (i) initialization of electron spin to its ground state in magnetic field; (ii) ac electrical control of the hyperfine coupling of the donor (A gate); (iii) on and off switching of exchange coupling between neighboring electrons ( $\mathrm{J}$ gate). Remarkably, our scheme only needs a subgigahertz exchange coupling, which can be satisfied for almost all donor pairs with separation $\sim 10 \mathrm{~nm}$. Hence it provides a practical way for generating the critical resource of multipartite entanglement of spins in silicon, which can tolerate the exchange oscillation problem and be realized within the current technology. Our scheme is a significant example of the conceptually new approach of dissipative quantum state preparation with the advantages of robustness and no need for accurate temporal controls as compared to conventional state preparation by coherent evolution, as the desired Dicke states all appear as the unique steady state under the various pumping scenarios. Numerical simulation with realistic parameters for ${ }^{31} \mathrm{P}$ donors shows that 
(a)

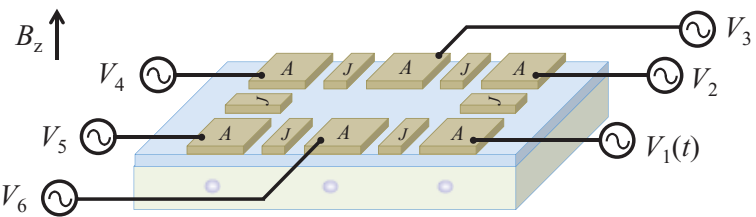

(b)

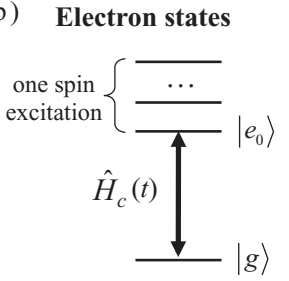

(c) $\left|e_{0}\right\rangle|J, M+1\rangle-$

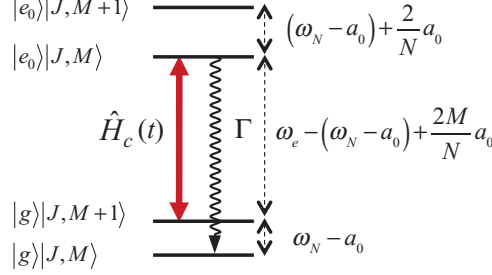

(d)

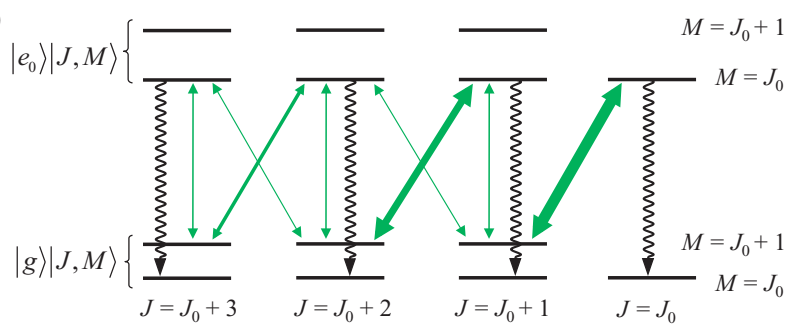

FIG. 1. (Color online) (a) Donor spins in silicon controlled by patterned electrodes. Pumping of nuclear spins is realized through ac voltage control of the A gates. (b) Energy level scheme for the exchange-coupled electrons in magnetic field along $z$ direction. The transition between the ground state $|g\rangle$ and a nondegenerate onespin-excitation state $\left|e_{0}\right\rangle$ is used. (c), (d) Pumping of nuclear spins by ac hyperfine coupling $\hat{H}_{c}$ (double-head arrows), assisted by the electron spin decay channel from $\left|e_{0}\right\rangle$ to $|g\rangle$ (wavy lines). The central frequency of $\hat{H}_{c}$ is tuned in resonance with the energy separation between $|g\rangle|J, M+1\rangle$ and $\left|e_{0}\right\rangle\left|J^{\prime}, M\right\rangle$, which is a quantity dependent on $M$. (c) When the ac voltage control uses the first scenario for phases (see text), population is deterministically transferred from the collective nuclear state $|J, M+1\rangle$ to $|J, M\rangle$. (d) When the ac voltage control uses the second scenario for phases (see text), population is transferred from $|J, M+1\rangle$ to $\left|J^{\prime}, M\right\rangle$, where $J^{\prime}-J=0$ or \pm 1 .

Dicke states of 10-20 qubits can be prepared with high fidelity in the presence of decoherence and unwanted dynamics. The scheme also applies to other donor systems with larger nuclear spins such as ${ }^{209} \mathrm{Bi}$ in silicon. ${ }^{27-29}$

\section{CONTROL SCHEME}

Figure 1(a) schematically illustrates the Kane architecture where shallow donors are embedded under patterned electrodes. The A gate tunes the hyperfine interaction $\hat{H}_{\mathrm{hf}}=$ $\sum_{n} a_{n} \hat{\boldsymbol{\sigma}}_{n} \cdot \hat{\boldsymbol{I}}_{n}$, with $\hat{\boldsymbol{\sigma}}_{n}$ and $\hat{\boldsymbol{I}}_{n}$ being the electron spin and nuclear spin of the $n$th donor, respectively. The coupling strength $a_{n}$ is proportional to the electron density at the donor nucleus site and hence is a function of the voltage applied to the A gate which pulls the electron wave function away from the nucleus. With each A gate independently controlled with voltage $V_{n}=V_{n, 0}+\delta V_{n}(t) \cos \left(\omega t+\phi_{n}\right)$, the hyperfine interaction becomes

$$
\hat{H}_{\mathrm{hf}}=\sum_{n} a_{n, 0} \hat{\boldsymbol{\sigma}}_{n} \cdot \hat{\boldsymbol{I}}_{n}+\frac{\partial a}{\partial V} \delta V_{n} \cos \left(\omega t+\phi_{n}\right) \hat{\boldsymbol{\sigma}}_{n} \cdot \hat{\boldsymbol{I}}_{n},
$$

where the first term is the static hyperfine coupling where $a_{n, 0} / 2 \pi \equiv a\left(V_{n, 0}\right) / 2 \pi \simeq 60 \mathrm{MHz}$, and the second term is the ac hyperfine coupling from the voltage modulation. In a strong magnetic field, the off-diagonal part of the static hyperfine is far off resonance; hence it only results in small shifts of energy levels which are negligible here. However, the off-diagonal part of the ac hyperfine can efficiently pump nuclear spin polarization when the modulation frequency $\omega$ is resonant to the electron-nuclear flip-flop transition. ${ }^{30-32}$

The exchange coupling between neighboring donor electrons is tuned by the $\mathrm{J}$ gate, which can separate the electrons. When the electrons are decoupled, nuclear spins of different donors can be independently pumped to the fully polarized state (i.e., nuclear spin initialization). When the electrons are coupled, nuclear spins can be cooperatively flipped by collective raising/lowering operators. We have previously proposed using such pumping to probabilistically prepare singlets of nuclear spins. ${ }^{33,34}$

In general, for coupled donor electrons in a uniform magnetic field along the $z$ direction, one can always find a nondegenerate one-spin-excitation eigenstate $\left|e_{0}\right\rangle \equiv \sum_{n} \alpha_{n} \hat{\sigma}_{n}^{+}|g\rangle$, with $|g\rangle \equiv|\downarrow \cdots \downarrow\rangle$ denoting the ground state. With the ac control frequency $\omega$ tuned to near resonance with the transition between $|g\rangle$ and $\left|e_{0}\right\rangle$, we can neglect all other far-detuned electron spin resonances. Dropping nonsecular terms, the full Hamiltonian of the electron nuclear spin system can then be written as $\hat{H}=\hat{H}_{0}+\hat{H}_{c}$,

$$
\begin{aligned}
\hat{H}_{0}= & \omega_{e}\left|e_{0}\right\rangle\left\langle e_{0}\right|+\omega_{N} \sum_{n} \hat{I}_{n}^{z} \\
& -|g\rangle\left\langle g\left|\sum_{n} a_{0} \hat{I}_{n}^{z}-\frac{N-2}{N}\right| e_{0}\right\rangle\left\langle e_{0}\right| \sum_{n} a_{0} \hat{I}_{n}^{z}, \\
\hat{H}_{c}= & e^{i \omega t}\left|e_{0}\right\rangle\langle g| \sum_{n} \Omega_{n}(t) e^{i \phi_{n}} \hat{I}_{n}^{-}+\text {H.c. }
\end{aligned}
$$

where $\omega_{e}$ is the electron resonance frequency between $\left|e_{0}\right\rangle$ and $|g\rangle$ in the magnetic field, $\omega_{N}$ is the nuclear Zeeman frequency, and $\Omega_{n} \equiv \frac{\partial a}{\partial V} \delta V_{n} \alpha_{n}$. The dc voltage of each A gate is set such that $a_{n, 0}=a_{0} . \hat{H}_{c}$ induces electron-nuclear flip flop, where the nuclear spin flip is in a cooperative form determined by the phases $\phi_{n}$ and amplitudes $\delta V_{n}$ of the ac controls. For simplicity, we consider hereafter a uniformly coupled Heisenberg ring with the eigenstate $\left|e_{0}\right\rangle=\sum_{n} \frac{1}{\sqrt{N}}(-1)^{n} \hat{\sigma}_{n}^{+}|g\rangle$, which is gapped from other one-spin-excitation states by $\Delta$ [Fig. 1(b)]. Then we have $\Omega_{n}(t)=(-1)^{n} \Omega(t)$, where $\Omega \equiv$ $\frac{1}{\sqrt{N}} \frac{\partial a}{\partial V} \delta V$.

We consider two scenarios for the phases $\phi_{n}$. In the first scenario, $(-)^{n} e^{i \phi_{n}}=1$ for all donors and hence the electronnuclear flip-flop term becomes $\hat{H}_{c}=\Omega(t)\left[e^{i \omega t}\left|e_{0}\right\rangle\langle g| \hat{J}^{-}+\right.$ H.c.]. Here $\hat{J}^{ \pm}=\hat{J}^{x} \pm i \hat{J}^{y}$, where $\hat{\boldsymbol{J}} \equiv \sum_{n} \hat{\boldsymbol{I}}_{n}$ is the collective spin of all nuclei. In the second scenario, $(-)^{n} e^{i \phi_{n}}=1$ for a set of donors (referred to as group A), while $(-)^{n} e^{i \phi_{n}}=-1$ for the rest (referred to as group $\mathrm{B})$, and hence the electron-nuclear flip-flop term is of the form $\hat{H}_{c}=\Omega(t)\left[e^{i \omega t}\left|e_{0}\right\rangle\langle g|\left(\hat{j}_{A}^{-}-\right.\right.$ $\left.\hat{j}_{B}^{-}\right)+$H.c.], where $\hat{j}_{A}$ and $\hat{j}_{B}$ are the collective spin of nuclei in group $A$ and group $B$, respectively, and $\hat{\boldsymbol{j}}_{A}+\hat{\boldsymbol{j}}_{B}=\hat{\boldsymbol{J}}$. Dicke states here refer to the common eigenstates of $\hat{J}^{2}$ and $\hat{J}^{z}$ with eigenvalues $J(J+1)$ and $M$, respectively. Consider first the subspace with $j_{A}=n_{A} I$ and $j_{B}=\left(N-n_{A}\right) I$, where $n_{A}$ and 
$N-n_{A}$ are the number of nuclei (of spin $I$ ) in group A and $\mathrm{B}$, respectively. $j_{A}$ and $j_{B}$ are conserved quantum numbers in the dynamics. Dicke states in this subspace can be uniquely specified as $|J, M\rangle$.

The first nontrivial element for preparing Dicke states is the deterministic population transfer from $|J, M+1\rangle$ to $|J, M\rangle$. Only the first scenario for the phase control is needed, where $\hat{H}_{c}$ conserves the quantum number $J$. We assume the population on the electron excited state $\left|e_{0}\right\rangle$ can be efficiently dumped to ground state with rate $\Gamma$, which can be realized, e.g., via the tunneling process between the donor and the SET island at low temperature. ${ }^{12,13}$ If the ac control frequency $\omega$ is in resonance with the transition $|g\rangle|J, M+1\rangle \leftrightarrow\left|e_{0}\right\rangle|J, M\rangle$, the population on $|g\rangle|J, M+1\rangle$ is pumped one-way to $|g\rangle|J, M\rangle$ via the Raman-type process [Fig. 1(c)]. For efficient initialization of the electron spin on the ground state, the electron Zeeman energy shall be large as compared to the temperature. With a typical temperature of $\sim 100 \mathrm{mK}$ used in the experiments, ${ }^{13}$ a magnetic field $\geqslant 0.3 \mathrm{~T}$ is required, which corresponds to ac modulation of the A-gate voltage in the frequency range $\geqslant 10 \mathrm{GHz}$.

There exists two unwanted couplings by the electronnuclear flip flop: (1) the coupling to other electron one-spinexcitation states which are detuned at least by $\Delta$ [Fig. 1(b)]; (2) the outcoupling of the final state $|g\rangle|J, M\rangle$ to $\left|e_{0}\right\rangle \mid J, M-$ $1\rangle$, which is also detuned by $\frac{2}{N} a_{0}$. For the latter, we note that for any pair of states $|g\rangle|J, M+1\rangle$ and $\left|e_{0}\right\rangle\left|J^{\prime}, M\right\rangle$ coupled by $\hat{H}_{c}$, the resonant frequency is $\omega_{e}-\omega_{N}+a_{0}+\frac{2 M}{N} a_{0}$, namely, the pumping is $M$ selective with proper choice of the ac control frequency. When the detuning $\delta \equiv \min \left\{\Delta, \frac{2}{N} a_{0}\right\} \gg$ $\Gamma$, these unwanted couplings cause a negligible leakage $\propto\left(\frac{\Gamma}{\delta}\right)^{2}$.

With the nuclear spins initialized on the unentangled polarized state $|J=N I, M=N I\rangle$, the above simple control can deterministically prepare all symmetric Dicke states. Examples for preparing symmetric Dicke states of $N=20$ ${ }^{31} \mathrm{P}$ nuclear spins are simulated using the master equation

$\dot{\rho}=i[\rho, \hat{H}]-\frac{\Gamma}{2}\left(\left|e_{0}\right\rangle\left\langle e_{0}|\rho+\rho| e_{0}\right\rangle\left\langle e_{0}|-2| g\right\rangle\left\langle e_{0}|\rho| e_{0}\right\rangle\langle g|\right)$.

The results are shown in Fig. 2. Clearly, the state preparation has the advantage that it is insensitive to the shape and area of the control pulse $\Omega(t)$. We take $a_{0} / 2 \pi=60 \mathrm{MHz}$, $\Gamma / 2 \pi=60 \mathrm{kHz}$, and assume the donor electron exchange of $0.5 \mathrm{GHz}$, which leads to $\Delta / 2 \pi \sim 25 \mathrm{MHz}$. The preparation takes a time $t_{p} \sim O(10) \mu$ s. In comparison, a single two-qubit nuclear spin gate mediated by such a small donor exchange would take $\sim 1 \mathrm{~ms} .{ }^{35}$ The probability to obtain each Dicke state is nearly unitary $(\geqslant 99.5 \%)$, and the imperfection is caused by the off-resonance couplings which may be further reduced by using smaller $\Omega$ with the cost of a longer preparation time. We further note that the reverse population transfer from $|J, M\rangle$ to $|J, M+1\rangle$ can be realized if global $\pi$ flips of nuclear spins are applied before and after the above pumping process with the central frequency set at $\omega=\omega_{e}-\omega_{N}+a_{0}-$ $\frac{2(M+1)}{N} a_{0}$.

The second nontrivial element is to realize the asymmetric Dicke state $\left|J=J_{0}, M=J_{0}\right\rangle$ with general values of $J_{0}$. This

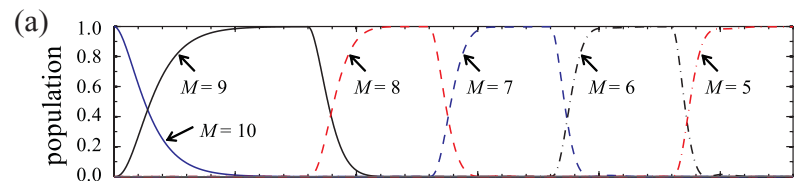

(b)
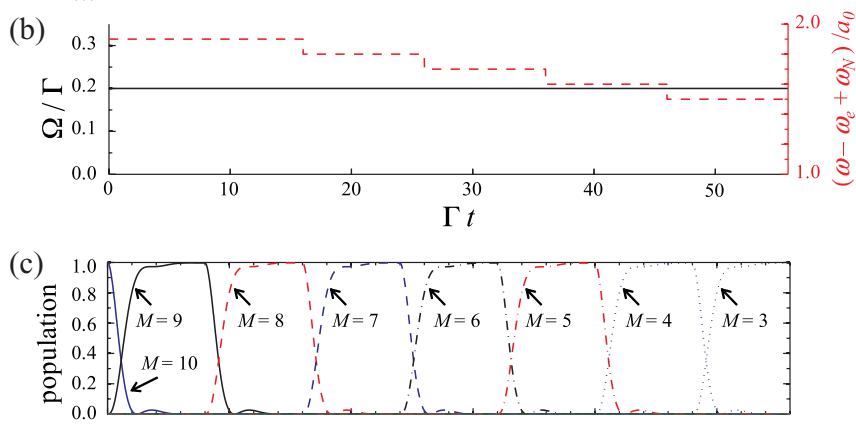

(d)

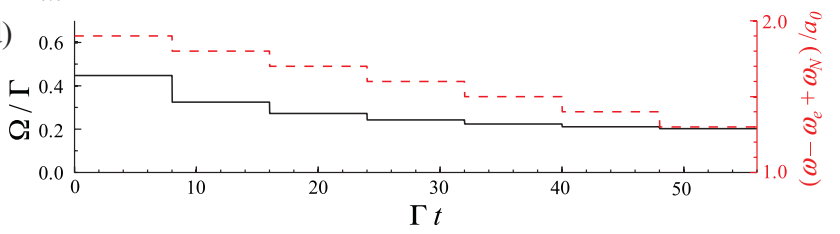

FIG. 2. (Color online) Preparation of the symmetric Dicke states of 20 nuclear spin qubits initially on the fully polarized state. For the numerical simulation in (a), (b), the amplitude $\Omega$ of the ac control is held as a constant while the central frequency $\omega$ steps down after a finite interval. Symmetric Dicke states $|J=10, M\rangle$ with different $M$ are obtained sequentially with a probability $\geqslant 99.8 \%$. In (c), (d), the control sequence $\Omega(t)$ is optimized for speed, and each Dicke state is obtained with a probability $\geqslant 99.5 \%$. $\Gamma / 2 \pi=60 \mathrm{kHz}$.

requires the quantum number $J$ to be changed, which needs the control with the second scenario for phases. As discovered by the authors in our earlier work, ${ }^{33,34}$ the inhomogeneous collective operator $\hat{j}_{A}^{-}-\hat{j}_{B}^{-}$couples $|J, M+1\rangle$ to the states $|J+\Delta J, M\rangle$ with the selection rule $\Delta J=0, \pm 1$. Hence $\hat{H}_{c}$ can resonantly drive the one-way pumping from $|J, M+1\rangle$ to $|J+\Delta J, M\rangle$ [see Fig. 1(d)]. By repeating the above pumping for $N I-J_{0}$ steps, the population can be transferred from the initial state $|J=N I, M=N I\rangle$ to the states $\mid J \geqslant$ $\left.J_{0}, M=J_{0}\right\rangle$ [see Fig. 3(a)]. If the reverse pumping from $\left|J, M=J_{0}\right\rangle$ to $\left|J, M=J_{0}+1\right\rangle$ (see proceeding paragraph) is also turned on, then all pathways from the initial state will end up at the desired target state $\left|J=J_{0}, M=J_{0}\right\rangle$ where the population gets trapped [see Fig. 3(a)]. This realizes the deterministic preparation of $\left|J=J_{0}, M=J_{0}\right\rangle$ with arbitrarily specified $J_{0}$. Figure $3(\mathrm{~b})$ shows the numerical simulation for preparing the Dicke state $\mid J=7, M=7, j_{A}=4, j_{B}=$ 6) of $20{ }^{31} \mathrm{P}$ nuclear spins, obtained with a probability of $99.5 \%$.

Combining these two elements, an arbitrary Dicke state can be deterministically prepared in the subspace defined by $j_{A}=n_{A} I$ and $j_{B}=\left(N-n_{A}\right) I$. Essentially, this is the realization of an arbitrary total spin eigenstate for the two collective spins $\hat{\boldsymbol{j}}_{A}$ and $\hat{\boldsymbol{j}}_{B}$. By concatenating this procedure, we can deterministically prepare any state in a complete Dicke-state basis for $N$ spins, denoted as $\left|J, M,\left\{j_{l, k}\right\}\right\rangle$, where $\left\{j_{l, k}\right\}$ are the collective spins of subsets [Fig. 4(a)]. The concatenated procedures are illustrated in Fig. 4(b). 

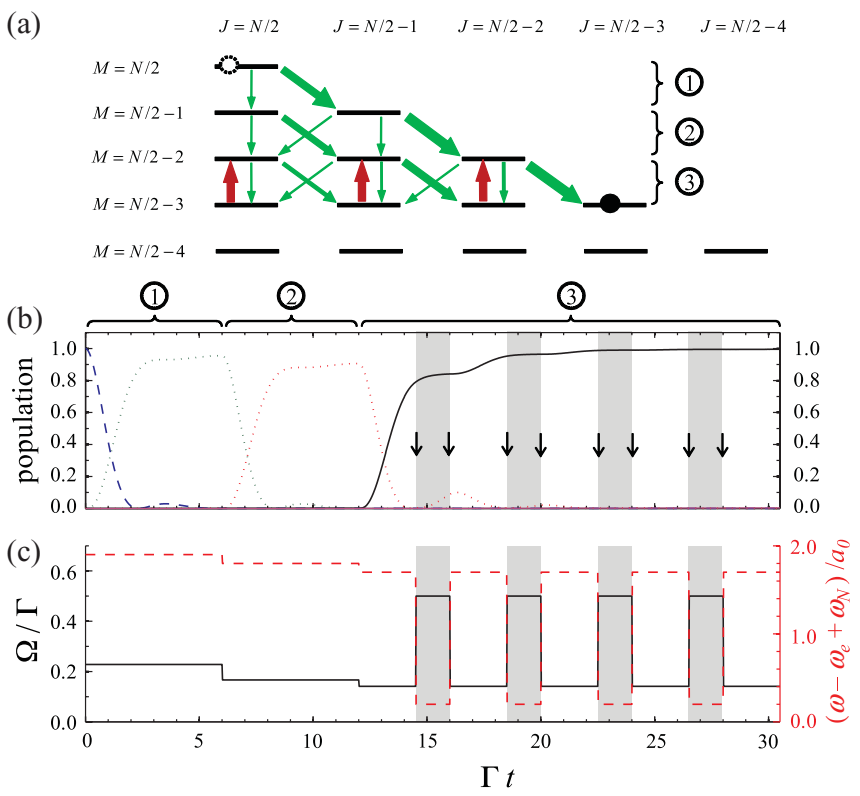

FIG. 3. (Color online) Preparation of asymmetric Dicke state $|J=7, M=7\rangle$ in the subspace $\left(j_{A}=4, j_{B}=6\right)$ for 20 nuclear spin qubits initially on the fully polarized state. (a) Population flows by the cooperative pumping. (b), (c) Numerical simulation of the state preparation. (b) Populations on the various Dicke states: $|J=10, M=10\rangle$ (dashed blue); $|J=9, M=9\rangle$ (dotted green); $|J=8, M=8\rangle$ (dotted red). The solid curve gives the population on the target state $|J=7, M=7\rangle$ (or its time reversal state in the shaded interval), and its final population is $99.5 \%$. (c) The magnitude $\Omega$ and central frequency $\omega$ for the ac tuning of hyperfine interaction. In the unshaded (shaded) interval, the first (second) scenario for the phases is applied (see text). The vertical arrows indicate timing of global $\pi$ flip of all nuclear spins. $\Gamma / 2 \pi=60 \mathrm{kHz}$.

\section{EFFECTS OF POSSIBLE IMPERFECTIONS}

Here we analyze the effects of various imperfections that may exist in realistic systems and give recipes on how to deal with these sources of errors.

The first cause of error is the decoherence of the spin qubits. Dicke states are immune to decoherence in a collective form, but independent nuclear spin decoherence can cause a leakage out of the desired subspace. For an $N$-qubit state, the leakage is $\sim t_{p} N \gamma$, where $\gamma$ is the nuclear spin decoherence rate and $t_{p}$ is the preparation time scale proportional to $\Gamma^{-1}$. The longest nuclear spin coherence time reported for $\mathrm{a}^{31} \mathrm{P}$ donor in silicon is $1.75 \mathrm{~s}^{1}{ }^{1}$ In Table I we show the performance of preparing several exemplary Dicke states in the presence of spin decoherence. Nuclear spin pure dephasing described by the Lindblad term $-\frac{\gamma}{2} \sum_{n}\left(\hat{I}_{n}^{z} \hat{I}_{n}^{z} \rho+\rho \hat{I}_{n}^{z} \hat{I}_{n}^{z}-2 \hat{I}_{n}^{z} \rho \hat{I}_{n}^{z}\right)$ is added to the master equation. The much slower nuclear spin relaxation process is neglected. ${ }^{36}$ Because of the short preparation time scale, there is no visible effect from the decoherence if we take $\gamma / 2 \pi=0.1 \mathrm{~Hz}$ from the state-of-the-art measurement. ${ }^{1}$ Even with the much exaggerated decoherence rate $\gamma / 2 \pi=$ $10 \mathrm{~Hz}$, the target states can still be obtained with high fidelity.

In realistic systems, there also exist various defects in the surroundings of the donors, such as the interface $\mathrm{P}_{b 0}$ centers. ${ }^{10,37-40}$ The interplay between $\mathrm{P}_{b 0}$ centers and (a)

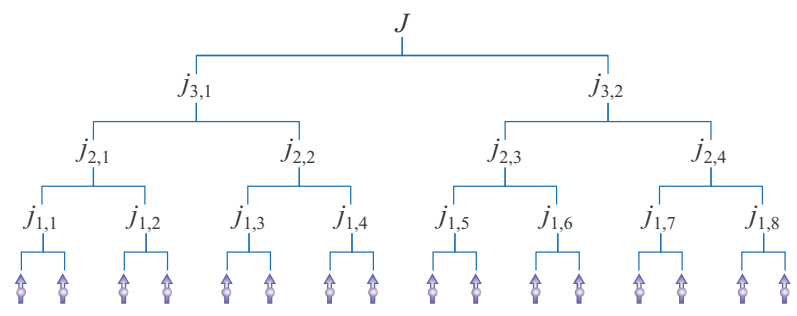

(b)

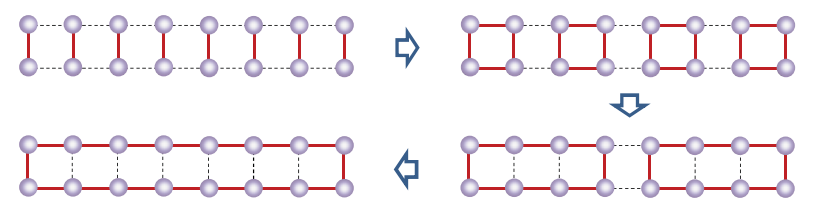

FIG. 4. (Color online) Preparation of Dicke states $\left|J, M,\left\{j_{l, k}\right\}\right\rangle$ which span a complete basis for the nuclear spin qubits. (a) The quantum numbers $j_{l, k}$ are the collective spin of subsets of qubits. (b) Concatenated preparation process. In step $l$, Dicke states are prepared in parallel in each $2^{l}$-qubit subset with the collective spin being the specified value $j_{l, k}$. The quantum numbers $j_{l<l_{0}, k}$ are all conserved in step $l_{0}$. Red solid (black dashed) lines indicate the donor electron exchange "on" (“off") when nuclear spins are pumped.

phosphorus electron spins is important in the presence of photoexcited electrons and holes. Such interplay can facilitate the electrical detection of paramagnetic resonance of the donor electron..$^{10,37-40}$ In the absence of illumination as in our scenario, at temperatures of $k_{B} T \ll g \mu B$, the $\mathrm{P}_{b 0}$ center as a midgap paramagnetic center can lead to statistical fluctuations of the electron-nuclear flip-flop resonances from donor to donor. Furthermore, there could be noises from the local electrostatic environment, e.g., possible charge traps in the gate oxide, ${ }^{13}$ which may affect the hyperfine coupling and the donor electron exchange as well. If the charge hopping is faster than the state preparation time scale, the effect of this dynamic noise shall be similar to the effect of spin decoherence analyzed above. It is also possible to interlace the pumping control with sequence of $\pi$ pulses applied to the spin qubits for dynamical decoupling from such noises. ${ }^{41}$ If charge hopping is much slower as compared to the state preparation time scale, the noises are static, which also results in the statistical fluctuation of the hyperfine coupling strength and exchange coupling strength.

To deal with such statistical fluctuations of system parameters, the system provides multifold individual tunability for each donor. The electron-nuclear flip-flop resonance depends on the static part of the hyperfine interaction [see Eq. (2a)], which can be independently controlled by each A gate. Thus, in the presence of inhomogeneous broadening of the electron-nuclear flip-flop resonances, the dc part of the A-gate voltage can tune the static part of the hyperfine coupling for compensating the inhomogeneity after calibration. As the excited state for the electron-nuclear flip flop has an intrinsic broadening of $\Gamma$, inhomogeneity in the flip-flop resonances is unimportant if it is less than this intrinsic level broadening.

The exchange couplings between donor electrons are generally inhomogeneous due to the exchange oscillation problem and the possible effects of the aforementioned charge noises. This inhomogeneity manifests as nonuniform amplitudes $\alpha_{n}$ 
TABLE I. Figure of merits for preparing Dicke states of 10 qubits in presence of decoherence. $\left|\psi_{1}\right\rangle \equiv|J=5, M=4\rangle$ is the W state. $\left|\psi_{2}\right\rangle \equiv|J=5, M=0\rangle$ is the symmetric Dicke state with the most spin excitations. $\left|\psi_{3}\right\rangle \equiv \mid J=2, M=$ $\left.2, j_{A}=\frac{5}{2}, j_{B}=\frac{5}{2}\right\rangle$ is an asymmetric Dicke state. $F \equiv\langle\psi|\rho| \psi\rangle$ is the fidelity of the density matrix with the target state at $t=t_{p}$. We take $\Gamma / 2 \pi=50 \mathrm{kHz}$, and $\Omega_{0}$ is the peak value of $\Omega(t)$ used.

\begin{tabular}{lrrcc}
\hline \hline Target & $\Omega_{0} / 2 \pi$ & $t_{p}$ & $F(\gamma / 2 \pi=0.1 \mathrm{~Hz})$ & $F(\gamma / 2 \pi=10 \mathrm{~Hz})$ \\
\hline$\left|\psi_{1}\right\rangle$ & $31.5 \mathrm{kHz}$ & $32 \mu \mathrm{s}$ & 0.999 & 0.998 \\
$\left|\psi_{2}\right\rangle$ & $31.5 \mathrm{kHz}$ & $108 \mu \mathrm{s}$ & 0.999 & 0.987 \\
$\left|\psi_{3}\right\rangle$ & $41 \mathrm{kHz}$ & $175 \mu \mathrm{s}$ & 0.998 & 0.982 \\
\hline \hline
\end{tabular}

in the electron excited state $\left|e_{0}\right\rangle \equiv \sum_{n} \alpha_{n} \hat{\sigma}_{n}^{+}|g\rangle$. We note that the pumping operators that cooperatively flip the nuclear spin qubits are of the form $\sum_{n} \frac{\partial a}{\partial V} \delta V_{n} \alpha_{n} e^{i \phi_{n}} \hat{I}_{n}^{-}$[see Eq. (2b)]. While J-gate voltages can tune the exchange couplings and hence the values of $\alpha_{n}$, the ac modulation strength $\delta V_{n}$ and phase $\phi_{n}$ can further compensate the remaining inhomogeneity in $\alpha_{n}$.

If only entanglement generation is of interest, our scheme can naturally cope with unknown systematic errors in the cooperative pumping. For controls aimed at the symmetric Dicke state $\left(\hat{J}^{-}\right)^{m}|J=N / 2, M=N / 2\rangle$, the nuclear spins may be flipped instead by $\hat{A}^{-} \equiv \sum_{n}\left(1+\eta_{n}\right) e^{i \theta_{n}} \hat{I}_{n}^{-}$, where $\theta_{n}$ and $\eta_{n}$ are the unknown phase and amplitude errors, respectively. In the presence of these errors, the steady state is still a definite pure state $\left(\hat{A}^{-}\right)^{m}|J=N / 2, M=N / 2\rangle$ with similar multipartite entanglement. This feature makes the requirement less stringent for implementing this scheme for entanglement generation.

Moreover, one advantage of our scheme is unwanted donor sites can be easily disconnected in the state preparation. There are two ways to do this. One is to turn off the coupling of such donor sites with others by the J-gate control. Even if the exchange interaction couples all neighboring donor electron spins into collective states, the set of nuclear spins being pumped can be further selected by the ac voltage controls. If the voltage applied to one of these donors has no ac component, its nuclear spin will not be pumped and is effectively decoupled from the rest in the preparation process. This advantage allows one to select out the set of donors where the parameters are relatively uniform, and it also facilitates the realization of the concatenated preparation process described in Fig. 4.

Finally, we give an estimate on the scale of the Dicke states that can be prepared using this approach. First, the detuning of the unwanted transitions is $\sim \frac{2}{N} a_{0}$, which scales inversely with the qubit number $N$. To suppress the unwanted transitions, we require $\frac{2}{N} a_{0} \gg \Gamma$. Second, the leakage due to qubit decoherence is $\sim t_{p} N \gamma$, while the preparation time $t_{p}$ is determined by the smaller one of the modulation amplitude of the hyperfine interaction $\Omega$ and the spin initialization rate $\Gamma$. In our simulation, we take $\Gamma \sim 50-60 \mathrm{kHz}$ and $\Omega \sim 30-40 \mathrm{kHz}$, both values in the range reported in experiments. ${ }^{10,11,13}$ Then $N$ shall not exceed 100 by the first requirement mentioned above. The upper bound for $N$ set by the second requirement depends on the qubit decoherence rate $\gamma$. For a given $\gamma$, the fidelity for state preparation in a block of $N$ qubits may be scaled from the numbers listed in Table I. For example, with the nuclear spin decoherence rate of $10 \mathrm{~Hz}\left(T_{2} \sim 15 \mathrm{~ms}\right)$, preparation of the asymmetric Dicke state $\left|\psi_{3}\right\rangle$ for 20 qubits can have a fidelity of over 0.96. Symmetric Dicke states may be prepared in a much larger scale, since they take much fewer steps by the cooperative pumping and $t_{p}$ is then considerably shorter.

\section{ACKNOWLEDGMENTS}

The work was supported by the Research Grant Council of Hong Kong under Grants No. HKU706711P and No. HKU8/CRF/11G. The authors acknowledge Andrea Morello and Wendi Li for helpful discussions. *wangyao@hkucc.hku.hk

${ }^{1}$ J. J. L. Morton, A. M. Tyryshkin, R. M. Brown, S. Shankar, B. W. Lovett, A. Ardavan, T. Schenkel, E. E. Haller, J. W. Ager, and S. A. Lyon, Nature (London) 455, 1085 (2008).

${ }^{2}$ A. M. Tyryshkin, S. A. Lyon, A. V. Astashkin, and A. M. Raitsimring, Phys. Rev. B 68, 193207 (2003).

${ }^{3}$ E. Abe, A. M. Tyryshkin, S. Tojo, J. J. L. Morton, W. M. Witzel, A. Fujimoto, J. W. Ager, E. E. Haller, J. Isoya, S. A. Lyon, M. L. W. Thewalt, and K. M. Itoh, Phys. Rev. B 82, 121201(R) (2010).

${ }^{4}$ B. E. Kane, Nature (London) 393, 133 (1998).

${ }^{5}$ R. Vrijen, E. Yablonovitch, K. Wang, H. W. Jiang, A. Balandin, V. Roychowdhury, T. Mor, and D. DiVincenzo, Phys. Rev. A 62, 012306 (2000).

${ }^{6}$ A. J. Skinner, M. E. Davenport, and B. E. Kane, Phys. Rev. Lett. 90, 087901 (2003).
${ }^{7}$ R. de Sousa, J. D. Delgado, and S. Das Sarma, Phys. Rev. A 70, 052304 (2004).

${ }^{8}$ J. J. L. Morton, e-print arXiv:0905.4008.

${ }^{9}$ D. N. Jamieson, C. Yang, T. Hopf, S. M. Hearne, C. I. Pakes, S. Prawer, M. Mitic, E. Gauja, S. E. Andresen, F. E. Hudson, A. S. Dzurak, and R. G. Clark, Appl. Phys. Lett. 86, 202101 (2005).

${ }^{10}$ L. Dreher, T. A. Hilker, A. Brandlmaier, S. T. B. Goennenwein, H. Huebl, M. Stutzmann, and M. S. Brandt, Phys. Rev. Lett. 106, 037601 (2011)

${ }^{11}$ F. R. Bradbury, A. M. Tyryshkin, G. Sabouret, J. Bokor, T. Schenkel, and S. A. Lyon, Phys. Rev. Lett. 97, 176404 (2006).

${ }^{12}$ A. Morello, C. C. Escott, H. Huebl, L. H. Willems van Beveren, L. C. L. Hollenberg, D. N. Jamieson, A. S. Dzurak, and R. G. Clark, Phys. Rev. B 80, 081307 (2009).

${ }^{13}$ A. Morello, J. J. Pla, F. A. Zwanenburg, K. W. Chan, K. Y. Tan, H. Huebl, M. Möttönen, C. D. Nugroho, C. Yang, J. A. van 
Donkelaar, A. D. C. Alves, D. N. Jamieson, C. C. Escott, L. C. L. Hollenberg, R. G. Clark, and A. S. Dzurak, Nature (London) 467, 687 (2010).

${ }^{14}$ S. Das Sarmaa, R. de Sousa, X. Hu, and B. Koiller, Solid State Commun. 133, 737 (2005).

${ }^{15}$ F. J. Ruess, L. Oberbeck, M. Y. Simmons, K. E. J. Goh, A. R. Hamilton, T. Hallam, S. R. Schofield, N. J. Curson, and R. G. Clark, Nano Lett. 4, 1969 (2004).

${ }^{16}$ H. Häffner, W. Hänsel, C. F. Roos, J. Benhelm, D. Chek-al-kar, M. Chwalla, T. Körber, U. D. Rapol, M. Riebe, P. O. Schmidt, C. Becher, O. Gühne, W. Dür, and R. Blatt, Nature (London) 438, 643 (2005).

${ }^{17}$ R. Prevedel, G. Cronenberg, M. S. Tame, M. Paternostro, P. Walther, M. S. Kim, and A. Zeilinger, Phys. Rev. Lett. 103, 020503 (2009).

${ }^{18}$ W. Wieczorek, R. Krischek, N. Kiesel, P. Michelberger, G. Tóth, and H. Weinfurter, Phys. Rev. Lett. 103, 020504 (2009).

${ }^{19}$ M. Eibl, N. Kiesel, M. Bourennane, C. Kurtsiefer, and H. Weinfurter, Phys. Rev. Lett. 92, 077901 (2004).

${ }^{20}$ L.-M. Duan and H. J. Kimble, Phys. Rev. Lett. 90, 253601 (2003).

${ }^{21}$ A. S. Sørensen and K. Mølmer, Phys. Rev. Lett. 91, 097905 (2003).

${ }^{22}$ J. K. Stockton, R. van Handel, and H. Mabuchi, Phys. Rev. A 70, 022106 (2004).

${ }^{23}$ C. Thiel, J. von Zanthier, T. Bastin, E. Solano, and G. S. Agarwal, Phys. Rev. Lett. 99, 193602 (2007).

${ }^{24}$ L. M. Duan, Phys. Rev. Lett. 107, 180502 (2011).

${ }^{25}$ M. Murao, D. Jonathan, M. B. Plenio, and V. Vedral, Phys. Rev. A 59, 156 (1999).
${ }^{26}$ J. Kempe, D. Bacon, D. A. Lidar, and K. B. Whaley, Phys. Rev. A 63, 042307 (2001).

${ }^{27}$ R. E. George, W. Witzel, H. Riemann, N. V. Abrosimov, N. Nötzel, M. L. W. Thewalt, and J. J. L. Morton, Phys. Rev. Lett. 105, 067601 (2010).

${ }^{28}$ M. H. Mohammady, G. W. Morley, and T. S. Monteiro, Phys. Rev. Lett. 105, 067602 (2010).

${ }^{29}$ G. W. Morley, M. Warner, A. M. Stoneham, P. T. Greenland, J. van Tol, C. W. M. Kay, and G. Aeppli, Nature Mater. 9, 725 (2010).

${ }^{30}$ M. S. Rudner and L. S. Levitov, Phys. Rev. Lett. 99, 246602 (2007).

${ }^{31}$ E. A. Laird, C. Barthel, E. I. Rashba, C. M. Marcus, M. P. Hanson, and A. C. Gossard, Phys. Rev. Lett. 99, 246601 (2007).

${ }^{32}$ E. I. Rashba, Phys. Rev. B 78, 195302 (2008).

${ }^{33}$ W. Yao, Phys. Rev. B 83, 201308 (2011).

${ }^{34}$ H. Y. Yu, Y. Luo, and W. Yao, Phys. Rev. A 84, 032337 (2011).

${ }^{35}$ C. D. Hill and H.-S. Goan, Phys. Rev. A 68, 012321 (2003).

${ }^{36}$ D. R. McCamey, J. V. Tol, G. W. Morley, and C. Boehme, Science 330, 1652 (2010).

${ }^{37}$ A. R. Stegner, C. Boehme, H. Huebl, M. Stutzmann, K. Lips, and M. S. Brandt, Nat. Phys. 2, 835 (2006).

${ }^{38}$ H. Huebl, F. Hoehne, B. Grolik, A. R. Stegner, M. Stutzmann, and M. S. Brandt, Phys. Rev. Lett. 100, 177602 (2008).

${ }^{39}$ G. W. Morley, D. R. McCamey, H. A. Seipel, L.-C. Brunel, J. van Tol, and C. Boehme, Phys. Rev. Lett. 101, 207602 (2008).

${ }^{40}$ H. Morishita, L. S. Vlasenko, H. Tanaka, K. Semba, K. Sawano, Y. Shiraki, M. Eto, and K. M. Itoh, Phys. Rev. B 80, 205206 (2009).

${ }^{41}$ Z. R. Gong and W. Yao (in preparation). 